\title{
Unlike the synchronous Plasmodium falciparum and $P$. chabaudi infection, the $P$. berghei and $P$. yoelii asynchronous infections are not affected by melatonin
}

\author{
Piero Bagnaresi' \\ Eduardo Alves' \\ Henrique Borges da Silva' \\ Sabrina Epiphanio² \\ Maria M Mota ${ }^{2}$ \\ Célia RS Garcia' \\ 'Departamento de Fisiologia, Instituto \\ de Biociências, Universidade de São \\ Paulo, São Paulo, Brazil; ' ${ }^{2}$ Unidade \\ de Malária, Instituto de Medicina \\ Molecular, Universidade de Lisboa, \\ Lisboa, Portugal
}

Correspondence: Célia Regina da Silva Garcia

Rua do Matão, travessa 14, n. 321. Cidade Universitária, São Paulo, Brazil 05508-090

$\mathrm{Tel}+55$ II 309। 7518

Fax +55 || 309| 8095

Email cgarcia@usp.br

\begin{abstract}
We have previously reported that Plasmodium chabaudi and P. falciparum sense the hormone melatonin and this could be responsible for the synchrony of malaria infection. In P. chabaudi and P. falciparum, melatonin induces calcium release from internal stores, and this response is abolished by $\mathrm{U} 73122$, a phospholipase $\mathrm{C}$ inhibitor, and luzindole, a melatoninreceptor competitive antagonist. Here we show that, in vitro, melatonin is not able to modulate cell cycle, nor to elicit an elevation in intracellular calcium concentration of the intraerythrocytic forms of $P$. berghei or $P$. yoelii, two rodent parasites that show an asynchrononous development in vivo. Interestingly, melatonin and its receptor do not seem to play a role during hepatic infection by $P$. berghei sporozoites either. These data strengthen the hypothesis that hostderived melatonin does not synchronize malaria infection caused by $P$. berghei and $P$. yoelii. Moreover, these data explain why infections by these parasites are asynchronous, contrary to what is observed in P. falciparum and P. chabaudi infections.
\end{abstract}

Keywords: malaria, calcium, melatonin, cell cycle, rhythm, sporozoite

\section{Introduction}

Malaria, caused by the parasite of genus Plasmodium, represents a major public health issue due to the growing resistance to current anti-malarial drugs. ${ }^{1}$ The World Health Organization (WHO) estimates that 300 to 500 million people are infected annually, and the number of deaths exceeds one million. The periodical fever peaks, which occur generally in 24-hour multiple intervals are the most striking trait of the malarial infection. This observation suggests that the erythrocyte rupture and reinvasion process is an extremely synchronized event. When a red blood cell (RBC) is infected by Plasmodium, their spectrin network is changed by the parasite. ${ }^{2}$ This RBC modification is only one of many operated by the parasite during its life cycle, due to, for example, intercellular protein trafficking. ${ }^{3}$

In 1929, Boyd $^{4}$ demonstrated that the life cycle of the chicken parasite $P$. cathemerium changed in accordance with changes in the light/dark cycles to which the host was submitted. In 1934, Taliaferro and Taliaferro ${ }^{5}$ delayed the schizogony of the monkey parasite $P$. brazilianum in 12 hours only by altering the host's photoperiod. This evidence stressed the importance of the photoperiod on the parasite's life cycle. In 1939, Stauber ${ }^{6}$ reported that incident light on the host's body surface or shone directly over the parasites does not alter the development of the infection. The light has to be perceived by the host for the synchronization signal to be delivered to the parasites in the bloodstream. 
In 1970, Hawking, ${ }^{7}$ who was studying $P$. vivax-infected patients, suggested that fever results from a burst in the number of merozoites in the host's bloodstream, and concluded that the parasites display a synchronous development to produce this population increase.

In 1976, Trager and Jensen ${ }^{8}$ successfully maintained $P$. falciparum in culture, and observed that the parasites lost their synchrony. This observation suggested an involvement of the host's physiology on the maintenance of the infection rhythm. The host's temperature was suggested to perform a role in this phenomenon, as there is a conspicuous period between the fever peaks. ${ }^{9}$ However, this possibility was rejected based on several lines of evidence, such as the variation of the schizogony times between different Plasmodium species. ${ }^{10,11}$

A periodicity is also observed during the sexual stage of Plasmodium's life cycle. This observation was initially made by Shah in 1934, who encountered a peak in $P$. cathemeruim gametocyte number at $1800 \mathrm{hrs}$. Similar results were reported by Gambrell ${ }^{12}$ and Hawking and collaborators. ${ }^{13}$ In 1970 , Hawking showed that production of gametocytes of the monkey parasite $P$. knowlesi depends on the host's circadian rhythm, increasing significantly during the night. This phenomenon has since been reported for several Plasmodium species. ${ }^{14}$ The appearance of the invertebrate-infective forms in the bloodstream at a time close to the feeding period the vector is a very important adaptive feature, which ensures the propagation of the infection. This cyclic and precise temporization of the appearance of gametocytes, coinciding with the vector's feeding pattern was called the "Hawking Phenomenon" by Garnham and Powers. ${ }^{15}$

Melatonin, a hormone secreted in a rhythmic fashion by the pineal gland, is a highly conserved molecule, as its presence can be observed in organisms ranging from archaebacterias to vertebrates. ${ }^{16,17}$

We have shown that the hormone melatonin is able to synchronize the life cycle of $P$. chabaudi and $P$. falciparum in vitro and that this effect is abolished by luzindole, a melatonin receptor antagonist. ${ }^{18}$ The synchronism is also lost in vivo in pinealectomized mice and upon injection of luzindole. Furthermore, synchronism in pinealectomized mice can be restored by melatonin administration.

The question then arises as to the evolutionary advantage for the parasites of cell cycle synchronization by host-produced melatonin. A hypothesis is that synchronous maturation might be a strategy to evade the host's immune system. ${ }^{18-20}$

As to the molecular mechanism of melatonin action in the parasites we have shown that melatonin can elicit an increase in intracellular calcium concentration in Plasmodium trophozoites. In addition, in P. falciparum, we have demonstrated that the melatonin-signaling pathway involves a complex crosstalk between $\mathrm{Ca}^{2+}$ and cAMP, ${ }^{21}$ and further activation of protein kinase A (PKA). Protein kinases are key components in Plasmodium-signaling pathways. ${ }^{22-24}$ Plasmodia genomes encode handling machinery for the both second messengers. ${ }^{25,26}$ Additionally, several reports support the importance of calcium signaling in parasites. ${ }^{27-36} \mathrm{Calcium}$ also plays a crucial role on invasion events, inducing proper apical alignment of the merozoite. ${ }^{37}$

In addition, Anopheles mosquito feeding habits occur during darkness, ${ }^{38-41}$ a period during which the levels of melatonin are the highest.

In this report we addressed the problem of the evolutionary role of synchronicity by comparing in vitro the effects of melatonin on cell cycle and $\mathrm{Ca}^{2+}$ levels in parasites that in vivo have a highly synchronous development ( $P$. chabaudi) with the strains $P$. berghei and $P$. yoelii that develop asynchronously in vivo. ${ }^{42-45}$

The data demonstrate that in vitro the asynchronous strain of $P$. berghei and $P$. yoelii melatonin is devoid of any effect on either $\mathrm{Ca}^{2+}$ signaling or cell cycle control on the blood stage and that melatonin does not produce any effect in $P$. berghei infection of mice and infected primary hepatocytes.

Here we present evidence that $P$. berghei and $P$. yoelii, both of which lead to asynchronous infections, do not respond to melatonin, strengthening the evidence that melatonin plays a major role in determining the rhythm of Plasmodium infection.

\section{Materials and methods}

\section{Parasites}

$P$. berghei NK65 and $P$. yoelii were maintained in BALB/c mice by infection passaging. The procedure for collecting blood and removing platelets has been described previously by Hotta and colleagues. ${ }^{46}$

P. berghei ANKA sporozoites were obtained from the salivary glands of infected Anopheles stenphensi mosquitoes and used to perform ex vivo and in vivo liver stage infection experiments.

\section{In vivo experiment with $P$. berghei}

Wistar rats, with a body weight of approximately $300 \mathrm{~g}$, where inoculated with $10^{7}$ erythrocytes infected with $P$. berghei NK65 parasites. The rats were maintained with food and water ad libitum, in a 12 hours light/12 hours dark 
photoperiodic regime. Every day, at ZT11, blood samples were collected from tail blood, to measure parasitemia by counting no less than 1000 cells in Giemsa-stained blood smears.

In hepatic stage assays 6-10 weeks old, male $\mathrm{C} 3 \mathrm{H}$ mice, which have a high physiological concentration of melatonin, ${ }^{47}$ were maintained in a 12 hours light/12 hours dark photoperiodic regime.

Luzindole treatment was performed by i.v. injection of $15 \mathrm{mg} / \mathrm{kg}$ of luzindole (Sigma-Aldrich, St Louis, MO) at midnight on three consecutive days. Mice were infected with 20.000 sporozoites at day 2 of luzindole treatment.

\section{Fluorescent $\mathrm{Ca}^{2+}$ determinations}

To obtain isolated parasites, $10^{8}$ infected $\mathrm{RBC}$ per $\mathrm{ml}$ were briefly treated with saponin $(10 \mathrm{mg} / \mathrm{ml})$ and washed twice in buffer A (116 mM NaCl, $5.4 \mathrm{mM} \mathrm{KCl,} 0.8 \mathrm{mM}$ $\mathrm{MgSO}_{4}, 5.5 \mathrm{mM}$ D-Glucose and $50 \mathrm{mM}$ MOPS, pH 7.2) and resuspended in the same buffer supplemented with $2.8 \mathrm{mM}$ probenecid (Sigma-Aldrich), an organic anion transport inhibitor. ${ }^{48}$ The cell suspension was then incubated for $50 \mathrm{~min}$ at $37^{\circ} \mathrm{C}$ with $6 \mu \mathrm{M}$ Fluo-3 AM (Molecular Probes, Carlsbad, CA) and washed three times with buffer A, for removal of extracellular probe. All the experiments and incubations were carried out in the presence of protease inhibitors: leupeptin, pepstatin A, antipain, chymostatin $(20 \mu \mathrm{g} / \mathrm{ml})$ and $0.5 \mathrm{mM}$ benzamidin.

Fluorescence was measured at $37^{\circ} \mathrm{C}$ with a Shimadzu RF-5301PC Spectrofluorimeter (Shimadzu, Kyoto, Japan), with an excitation wavelength of $505 \pm 5 \mathrm{~nm}$ and an emission wavelength of $530 \pm 5 \mathrm{~nm}$. Calcium concentration was assessed as described in Hotta and colleagues. ${ }^{46}$

\section{In vitro experiments}

Parasites were maintained in RPMI 1640 with $25 \mathrm{mM}$ HEPES (4-(2-hydroxyethyl)-1-piperazineethanesulfonic acid), supplemented with 50\% FCS. Melatonin was added at different concentrations, and incubated for 18 hours. Analyses of Giemsa-stained smears were performed, counting no less than 1000 cells per smear for parasitemia and life form distribution assessment. Triplicate smears were prepared for each experimental condition. Results are presented as the mean of three independent experiments.

\section{Infection quantification ex vivo}

Mouse primary hepatocytes were isolated by perfusion of mouse liver lobules with liver perfusion medium (Gibco/ Invitrogen, Carlsbad, CA) and purified using a $1.12 \mathrm{~g} / \mathrm{ml}$;
$1.08 \mathrm{~g} / \mathrm{ml}$ and $1.06 \mathrm{~g} / \mathrm{ml}$ Percoll gradient. Cells $\left(5 \times 10^{4} \mathrm{per}\right.$ well) were cultured in William's E medium containing 4\% FCS, 1\% penicillin/streptomicin, in Lab-Tek ${ }^{\mathrm{TM}}$ chamber slides $\left(\mathrm{Nunc}^{\mathrm{TM}}\right)$. Hepatocytes were maintained in culture at $37{ }^{\circ} \mathrm{C}$ and $5 \% \mathrm{CO}_{2}$.

Primary hepatocytes were incubated with $400 \mathrm{nM}$ melatonin for 30 minutes and infected with $20.000 P$. berghei sporozoites.

Infection was determined 45 hours after sporozoite addition by counting the number of exoerythrocytic forms (EEFs) on cells fixed with ice-cold methanol for 10 minutes and stained with the mouse monoclonal antibody $2 \mathrm{E} 6{ }^{49}$ and an AlexaFluor488 labeled goat anti-mouse secondary antibody (Molecular Probes/Invitrogen).

\section{Infection quantification by qRT-PCR}

The determination of liver parasite load in vivo, was performed according to Bruna-Romero and colleagues. ${ }^{50}$ Livers were collected and homogenized in denaturing solution (4 M guanidine thiocyanate, $25 \mathrm{mM}$ sodium citrate $\mathrm{pH} 7,0.5 \%$ sarcosyl and $0.7 \% \beta$ Mercaptoethanol in diethylpyrocarbonate [DEPC]-treated water), $40 \mathrm{~h}$ after sporozoite injection. Total RNA was extracted using Qiagen's RNeasy Mini kit, following the manufacturer's instructions. RNA for infection measurements was converted into cDNA using Roche's Transcriptor First Strand cDNA Synthesis kit, according to the manufacturer's protocol. The quantitative reverse transcription polymerase chain reactions (qRT-PCRs) were carried out using Applied Biosystems' Power SYBR Green PCR Master Mix and were performed according to the maunufacturer's instructions on an ABI Prism 7000 system (Applied Biosystems Inc., Foster City, CA). PbA-specific primer sequences were 5'-AAG CAT TAA ATA AAG CGA ATA CAT CCTTAC $-3^{\prime}$ and $5^{\prime}$-GGA GAT TGG TTT TGA CGT TTA TGT G $-3^{\prime}$.

\section{Results $\left[\mathrm{Ca}^{2+}\right]_{i}$ pools in $P$. berghei and $P$. yoelii}

A large number of cellular events in both low and high eukaryotes employ $\mathrm{Ca}^{2+}$-based signaling pathways. Extensive work on $\mathrm{Ca}^{2+}$ homeostasis and signaling has provided evidence for the major role of the endoplasmic reticulum in these processes as well of the participation of other organelles such as mitochondria, lisossomes and Golgi in $\mathrm{Ca}^{2+}$ storage in mammalian cells. ${ }^{51}$

P. berghei possesses calcium-handling mechanisms, such as the $\mathrm{Ca}^{2+}$-ATPases of endoplasmic reticulum (SERCA), sensitive endoplasmic reticulum (ER)-like pool and acidic pools. 
To investigate the role of intracellular $\mathrm{Ca}^{2+}$ pools in these parasites we have isolated $P$. berghei from red blood cells and loaded the parasites with Fluo-3 AM calcium dye. Figure 1a shows the effect of addition of $5 \mu \mathrm{M}$ thapsigargin (THG),
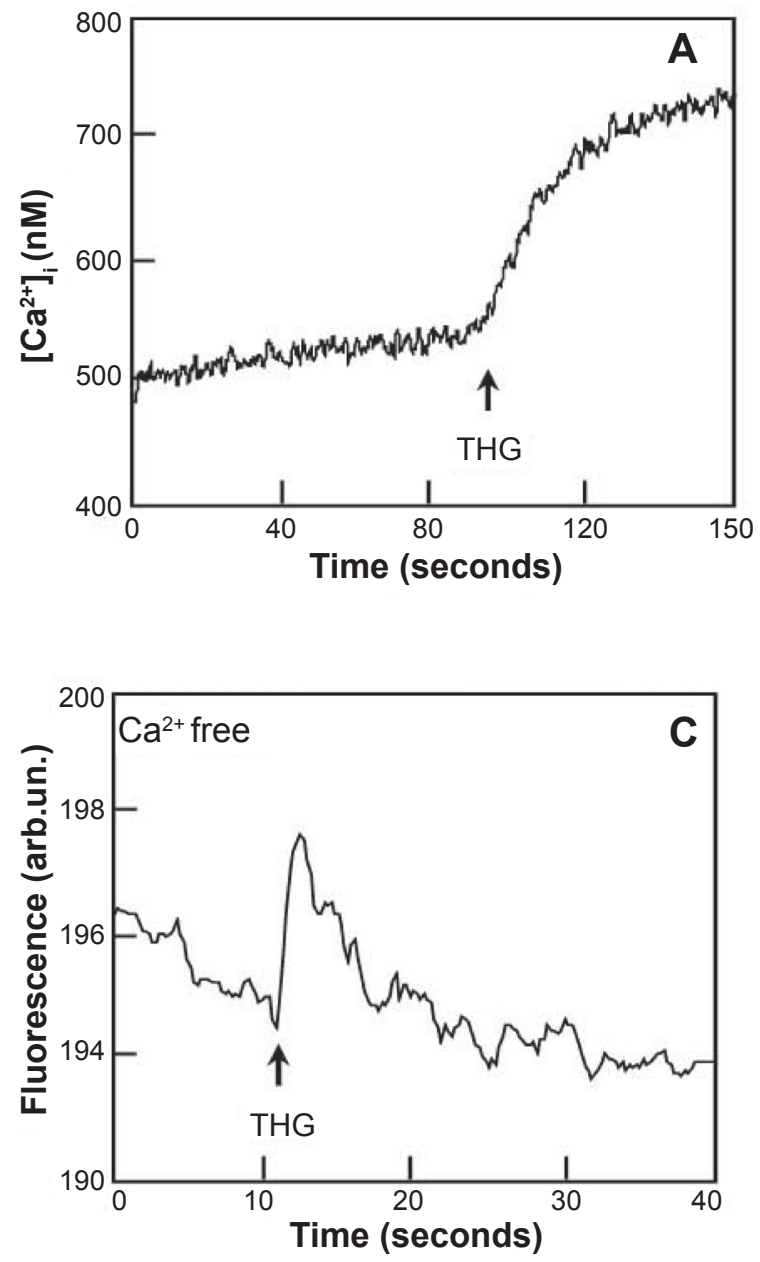

a SERCA inhibitor, ${ }^{52,53}$ on isolated $P$. berghei parasites. THG promotes an increase in the cytosolic calcium concentration, $(205 \pm 32.85 \mathrm{nM})$ thus confirming that these cells can store the $\mathrm{Ca}^{2+}$ ion in the ER. We further investigate whether acidic
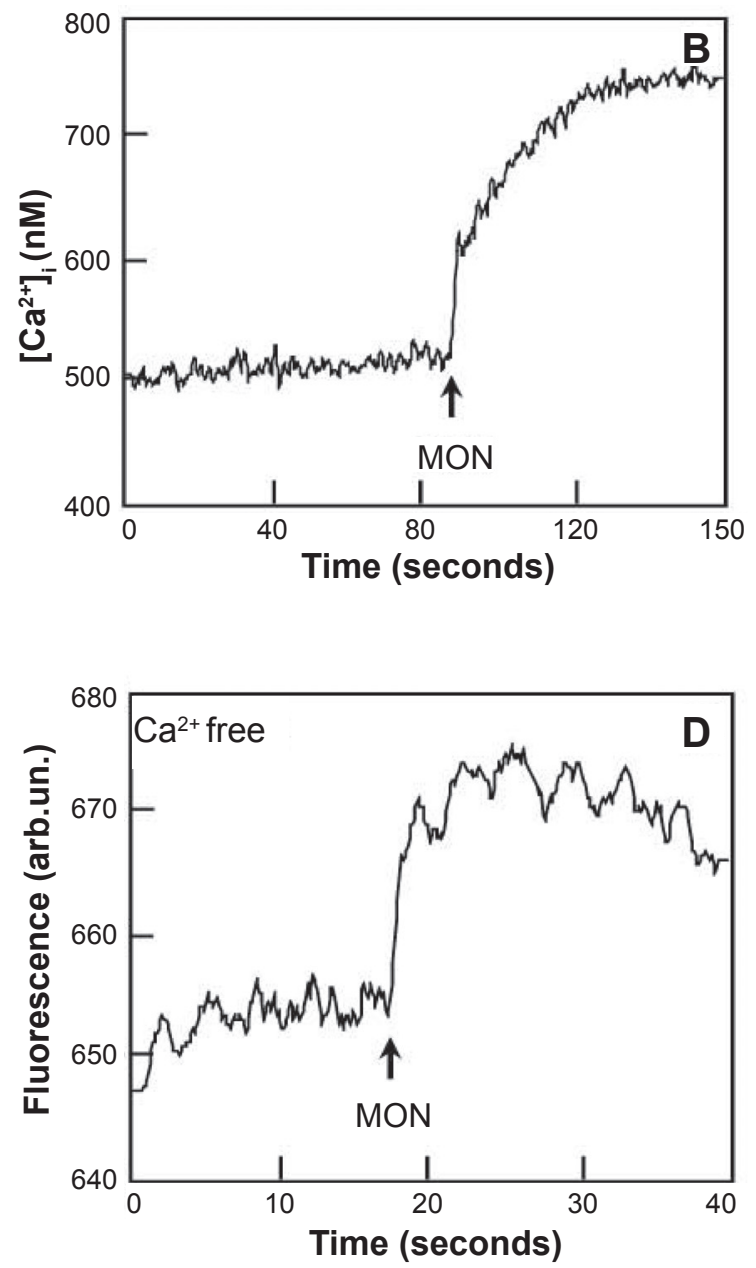

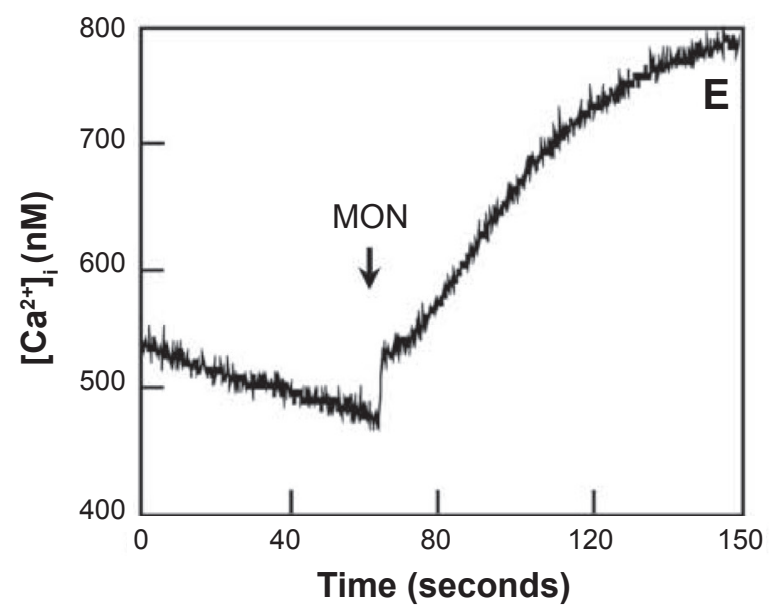

Figure I Calcium mobilization in Fluo-3 labeled P. berghei-isolated parasites. A) Addition of thapsigargin (THG, $5 \mu$ M) in I mM Calcium medium. B) Addition of $25 \mu$ M monensin (MON) to medium containing I mM Calcium. C) Addition of $5 \mu$ MTHG to calcium-free medium. D) Addition of $25 \mu \mathrm{M}$ MON to calcium-free medium. E) Addition of $20 \mu \mathrm{M}$ MON to $P$. yoelii parasites, in I mM calcium medium. 
pools could also play a role on $\mathrm{Ca}^{2+}$ homeostasis in $P$. berghei. Figure $1 \mathrm{~b}$ shows that monensin, a $\mathrm{Na}^{+} / \mathrm{H}^{+}$ionophore $(25 \mu \mathrm{M})$ elicits an $265 \pm 30.96 \mathrm{nM}$ increase in $\left[\mathrm{Ca}^{2+}\right]_{\mathrm{i}}$ of $P$. berghei.

$P$. yoelii also possesses mechanisms to handle calcium. Addition of THG to isolated parasites results in a $248 \pm 52.61 \mathrm{nM}$ $(\mathrm{n}=8)\left[\mathrm{Ca}^{2+}\right]_{\mathrm{i}}$ elevation (data not shown), showing that the ER is able to participate in calcium homeostasis. Acidic pools are also present and can be mobilized, as addition of monensin $(20 \mu \mathrm{M})$ promotes a $291.4 \pm 65.70 \mathrm{nM}\left[\mathrm{Ca}^{2+}\right]_{\mathrm{i}}$ increase (Figure 1E).

\section{Melatonin does not elicit an increase in $\mathrm{Ca}^{2+}$ concentration in P. berghei \\ and P. yoelii}

In order to further analyze the importance of melatonin in the control of Plasmodia cell cycle, we sought to investigate its effects on $P$. berghei, a species of Plasmodium that, unlike the vast majority of mammalian Plasmodium species, has an asynchronous development in the live mouse.

The simplest in vitro test to address the sensitivity of $P$. berghei and $P$. yoelii to melatonin is to determine whether the hormone can increase the cytoplasmic $\mathrm{Ca}^{2+}$ concentration, a well established early event caused by melatonin in $P$. chabaudi and $P$. falciparum. Figure 2 shows that addition of up to $20 \mu \mathrm{M}$ melatonin did not lead to an increase in $\mathrm{Ca}^{2+}$ concentration in P. berghei or P. yoelii, regardless of whether calcium was present or not in the medium. In addition, the effect of melatonin on P.yoelii is shown Figures 2C and D.
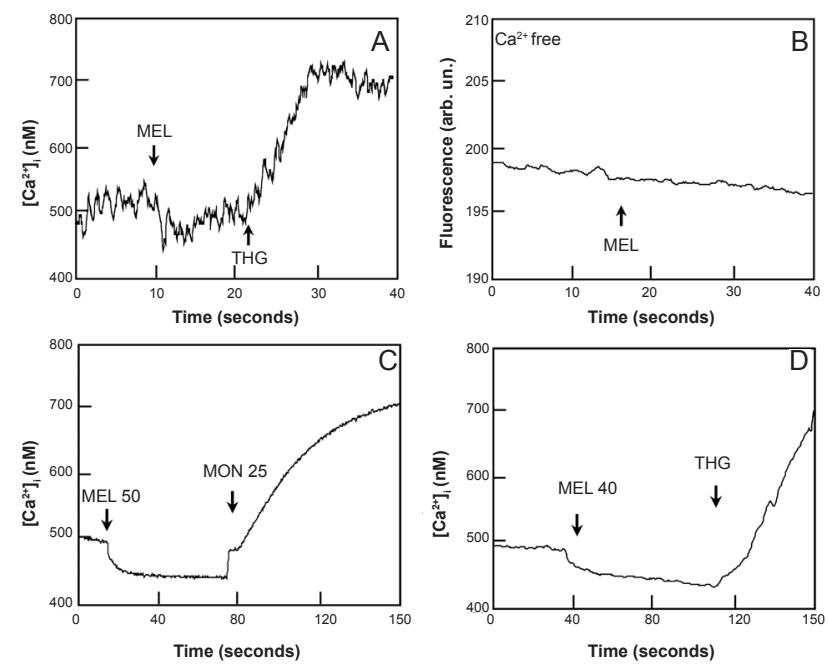

Figure 2 Effects of melatonin addition in isolated, Fluo-3 labeled, $P$. berghei and $P$. yoelii parasites. A) Addition of $20 \mu \mathrm{M}$ melatonin (MEL) to $P$. berghei in medium containing I mM calcium. B)Addition of $20 \mu \mathrm{M}$ MEL to P. berghei in calcium-free medium. C) Addition of $40 \mu \mathrm{M}$ melatonin (MEL 40) followed by $25 \mu \mathrm{M}$ monensin to $P$. yoelii in medium containing I mM calcium. D) Addition of $50 \mu$ M melatonin (MEL 50) followed by THG $(5 \mu \mathrm{M})$ to $P$. yoelii in medium containing I $\mathrm{mM}$ calcium. These experiments show that melatonin was not able to elicit a calcium response on these parasites.
The experiment shown in Figure 2 (panel a) shows that, similarly to what found in other Plasmodia, if after melatonin $P$. berghei are treated with thapsigargin (THG), an inhibitor of the sarco-endoplasmic reticulum ATPase, ${ }^{52,53}$ a clear increase in $\left[\mathrm{Ca}^{2+}\right]_{\mathrm{i}},(205 \pm 32.85 \mathrm{nM}, \mathrm{n}=3)$ is observed. In accordance with these results, panel $b$ shows that monensin, a Na $\mathrm{Na}^{+} / \mathrm{H}^{+}$ ionophore, that can induce the release of $\mathrm{Ca}^{2+}$ from an acidic pool in other Plasmodia strains, also elicits a strong increase in $\left[\mathrm{Ca}^{2+}\right]_{\mathrm{i}}, 265 \pm 30.96 \mathrm{nMn}=3$ in P. berghei. P. yoelii also lacks response for melatonin, while still have calcium pools that are capable of mobilization (Figures $2 \mathrm{C}$ and 2D). Taken together, these results most likely reflect the absence of a melatonin receptor coupled to $\mathrm{Ca}^{2+}$ mobilization rather than a unique characteristic of $P$. berghei or $P$. yoelii $\mathrm{Ca}^{2+}$ homeostasis.

\section{Melatonin does not interfere with $P$. berghei and $P$. yoelii life cycle}

Melatonin receptors might couple to other signaling pathways (eg, cAMP). In order to test whether in P. berghei melatonin could affect the cell cycle through a $\mathrm{Ca}^{2+}$-independent mechanism, we tested whether the hormone could synchronize in vitro $P$. berghei life cycle as is the case with $P$. chabaudi and $P$. falciparum. ${ }^{18,20,21}$ Again, hormone concentrations up to 100 -fold higher than those capable of strongly affecting Plasmodia development in the other strains were totally ineffective in synchronizing either $P$. berghei's or $P$. yoelii's life cycle (Figure 4).

$P$. berghei can infect both rats and mice. In C57BL/6 mice, $P$.berghei primarily causes a severe syndrome known as cerebral malaria. ${ }^{54}$ We thus checked whether the lack of in vitro sensitivity of $P$. berghei may be due to an artifact of these artificial culture conditions or of the specificity of the disease in mice. Wistar rats were inoculated with $10^{7}$ $P$. berghei-infected erythrocytes and the distribution of life forms on the fifth day after infection was investigated in blood smears. Figure 5 shows that also in rats $P$. berghei does not display a synchronous development. Indeed the percentage of rings and trophozoites are very similar (unlike in the mouse infected with $P$. chabaudi where trophozoites largely predominate at this time). Schizonts are hardly observed in infected rats as already reported by Desowitz and Barnwell presumably due to sequestration of the $\mathrm{RBC}$-containing parasites at this stage in the microvasculature. ${ }^{42}$

\section{Melatonin does not modify the $P$. berghei liver infection load}

The inhibition of melatonin receptor does not affect the parasite load in the livers of mice infected with $P$. berghei 

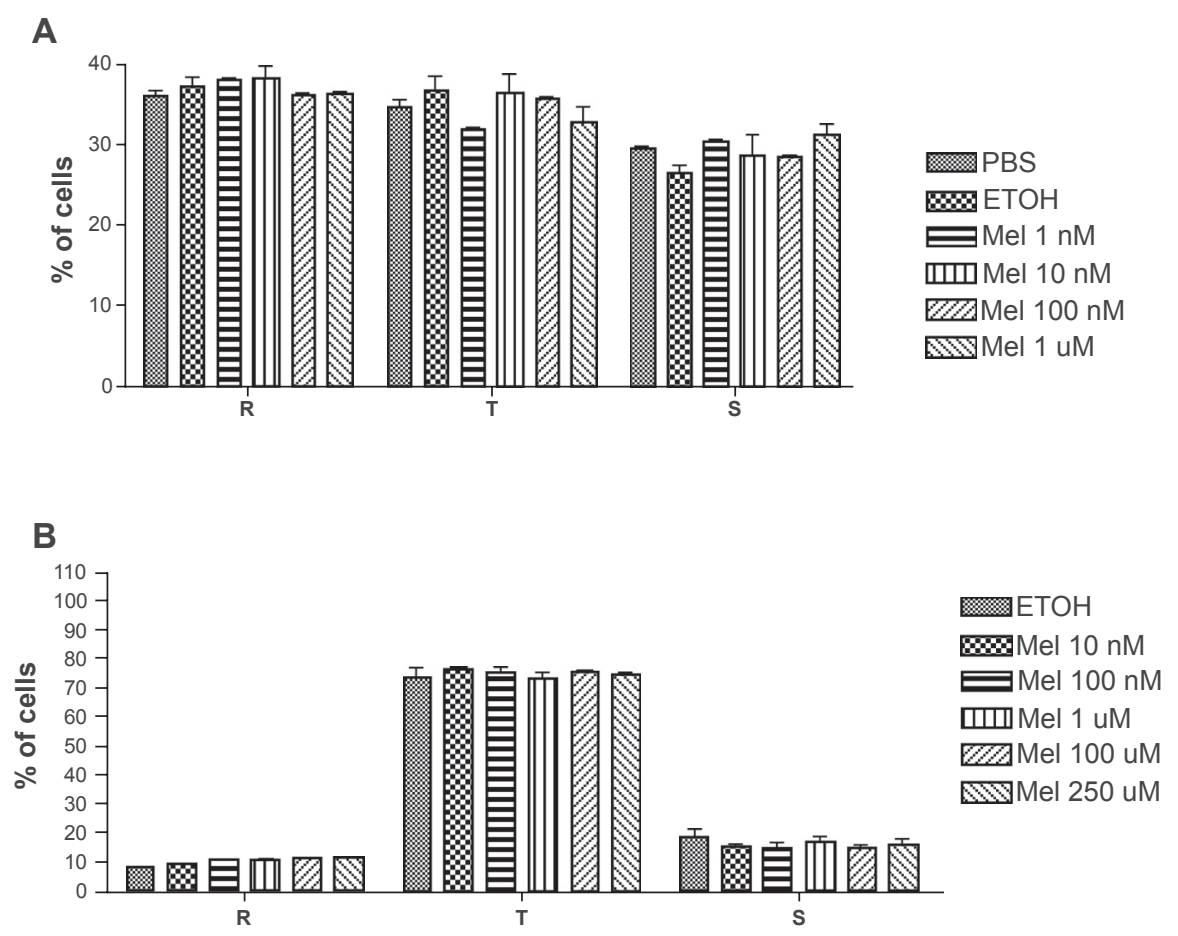

Figure 3 In vitro culture of $P$. berghei A) and P. yoelii B) incubated with different melatonin concentrations. The figure shows the distribution of $P$. berghei and $P$. yoelii life forms after 18 or 13 hours incubation, respectively, with different melatonin concentrations ( $\mathrm{nM}, 10 \mathrm{nM}, 100 \mathrm{nM}$ e I $\mu$ M). There are no statistical differences in the distribution. The results are presented as the mean of three independent experiments.
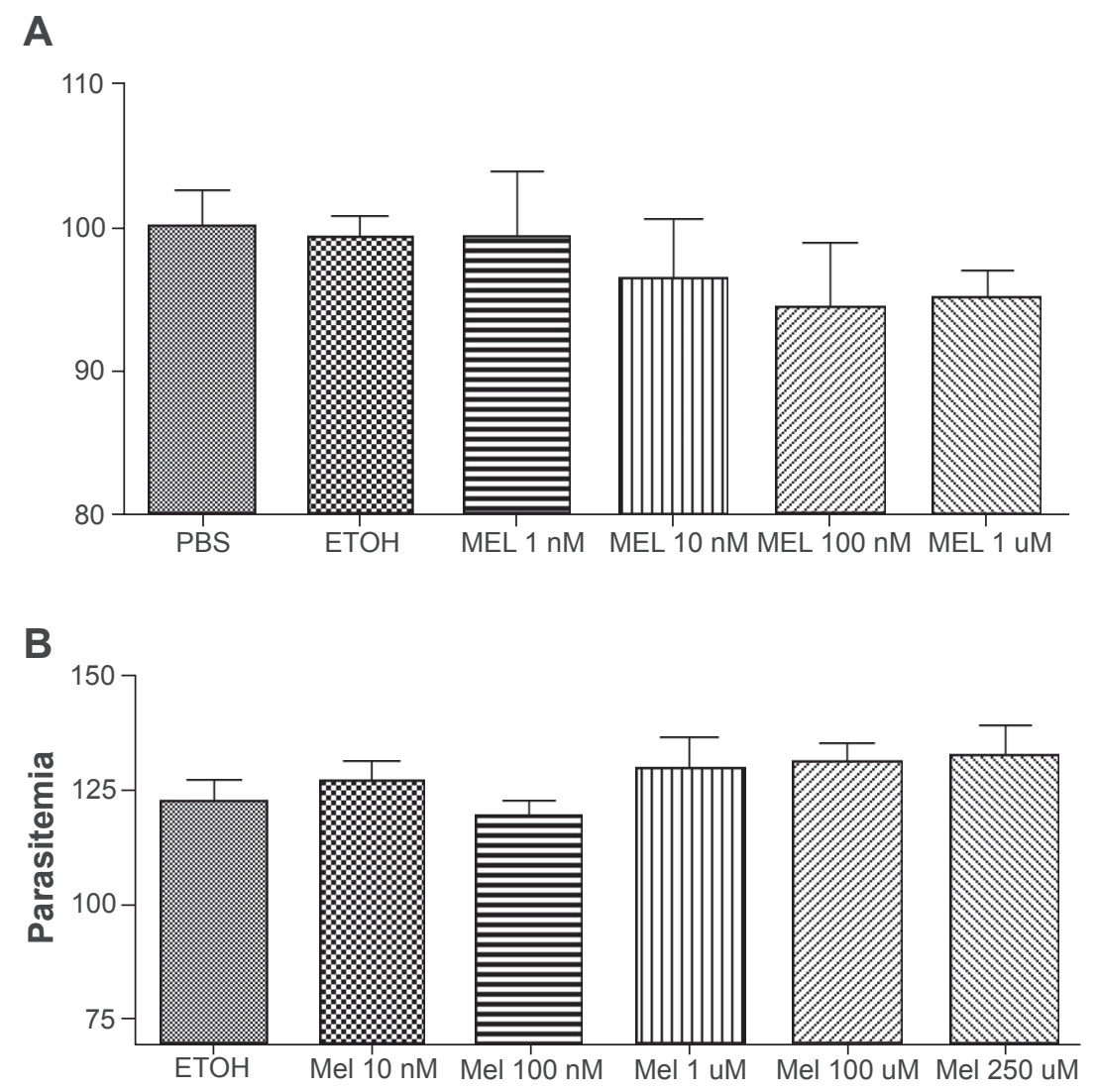

Figure 4 In vitro culture of $P$. berghei A) and $P$. yoelii B) incubated with different melatonin concentrations. The figure shows $P$. berghei- and $P$.yoelii-infected red blood cells (iRBC) after 18 or 13 hours incubation, respectively, melatonin concentrations ( $I \mathrm{nM}, 10 \mathrm{nM}, 100 \mathrm{nM}$ and I $\mu \mathrm{M})$. There are no statistical differences in the number of iRBC. The results are presented as the mean of three independent experiments. 


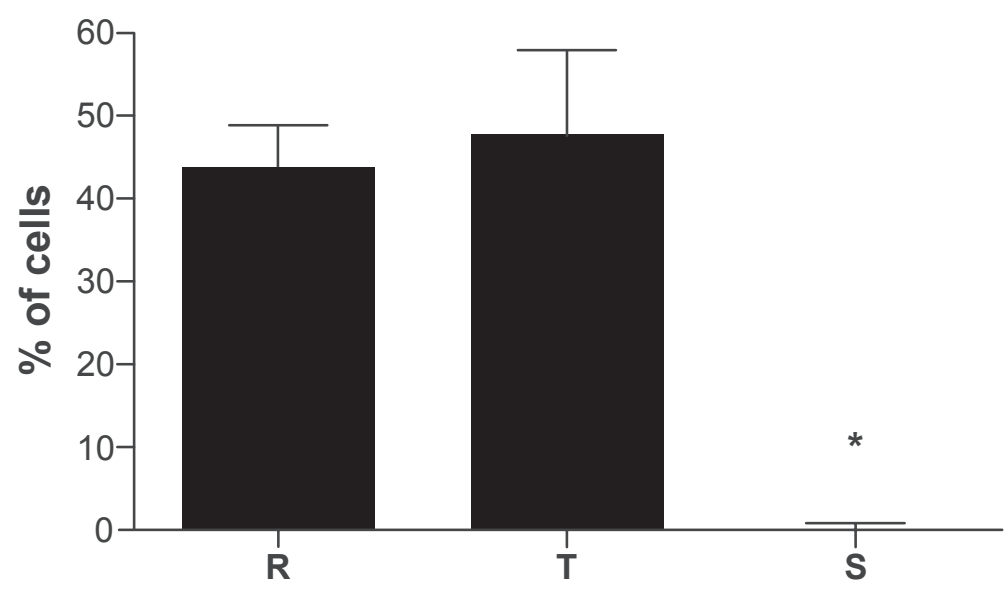

Figure 5 Distribution of $P$. berghei in infected Wistar rats, five days after inoculation of $10^{7}$ infected erythrocytes.

Notes: To assess life forms distributions, no less than 1000 cells were counted in Giemsa-stained smears. No statistical differences were observed between the percentage of rings and trophozoites. Schizonts were not present in peripheral bloodstream due to microvasculature sequestration.

Abbreviations: $\mathrm{R}$, rings; $\mathrm{T}$, trophozoites; $\mathrm{S}$, schizonts.

ANKA sporozoites. In addition, we did not observe a significant difference between the $P$. berghei infection levels of melatonin-treated mouse primary hepatocytes and that of control hepatocytes.

We have also considered the possibility that other stages of $P$. berghei might sense melatonin. Addition of melatonin to $P$. berghei gametocyte is not able to elicit an increase of calcium levels (Bilker O, personal communication) as is the case when xanthurenic acid is added. ${ }^{55}$

\section{Discussion}

The spectrofluorimetry results obtained show that $P$. berghei and $P$.yoelii display mechanisms that sustain the $\left[\mathrm{Ca}^{2+}\right]_{i}$ against an extracellular calcium concentration in the milimolar range. By using isolated parasites loaded with fluorescent dyes we showed here that the endoplasmatic reticulum plays a role in the $\left[\mathrm{Ca}^{2+}\right]_{i}$ maintenance. The storage of the calcium ion is mediated by a SERCA, since thapsigargin inhibits this enzyme, and promotes and $\left[\mathrm{Ca}^{2+}\right]_{i}$ increase. The experiments involving the $\mathrm{Na}^{+} / \mathrm{H}^{+}$ionophore monensin have shown that an acidic pool also participates in calcium homeostasis, as the ionophore also elicits an $\left[\mathrm{Ca}^{2+}\right]_{i}$ increase. The presence of intracellular calcium pools in $P$. berghei was previously demonstrated by Marchesini and colleagues. ${ }^{56}$

In 2000, Hotta and colleagues showed that melatonin could mobilize calcium from internal stores in isolated $P$. chabaudi and that this hormone is responsible for synchronization of the infection. The use of the phospholipase $\mathrm{C}$ inhibitor U73122 or of the melatonin receptor competitive antagonist luzindole abolished the melatonin-mediated calcium response, suggesting that a calcium pathway is involved in the transduction of the hormone signal. This pattern is also observed in P. falciparum, as reported by Beraldo and colleagues $^{21}$ using isolated parasites and infected RBCs.

Strikingly, melatonin does not induce an elevation in $\left[\mathrm{Ca}^{2+}\right]_{c}$ of $P$. berghei or $P$. yoelii, both of which lead to an
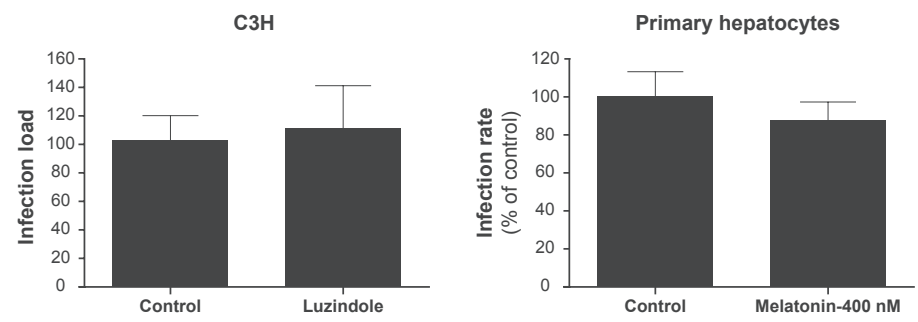

Figure 6 A) Effect of inhibition of melatonin receptor in $\mathrm{C} 3 \mathrm{H}$ mice infected by P. berghei sporozoites. Liver infection load was measured by qRT-PCR analysis of $P$. berghei $18 \mathrm{~S}$ rRNA in liver extracts taken $40 \mathrm{~h}$ after sporozoite i.v. injection, and plotted as a percentage of the mean of negative control samples. The plot represents three independent experiments $(n=18)$. No statistical significances were observed. B) Effect of $400 \mathrm{nM}$ melatonin on infection of mouse primary hepatocytes by P. berghei sporozoites. Infection rates were calculated for each sample well as the number of EEFs, plotted as a percentage relative to the mean of negative control samples. Results are expressed as the mean \pm SD of triplicate in three independent experiments.

Abbreviations: EEFs, exoerythrocytic forms; qRT-PCR, quantitative reverse transcriptase polymerase chain reaction; SD, standard deviation. 
unsynchronized infection. This observation prompted the question of whether melatonin was able to synchronize these infections, as we previously reported to be the case with both $P$. chabaudi and the human malaria parasite $P$. falciparum. To evaluate whether, despite not promoting calcium mobilization, melatonin was able to synchronize $P$. berghei and $P$. yoelii, we incubated parasites with various melatonin concentrations. The analysis of giemsa-stained smears has shown that the hormone was not able to synchronize the infection of these rodent parasites at a maximum concentration of $1 \mu \mathrm{M}$ for $P$. berghei, and $250 \mu \mathrm{M}$ for $P$. yoelii, in contrast to that is found in $P$. chabaudi, whose cell cycle can be modulated by $10 \mathrm{nM}$ of melatonin. ${ }^{18}$

Here we show that melatonin does not elicit a calcium response nor does it affect the distribution of $P$. berghei and $P$. yoelii life forms, which display an unsynchronized infection in vivo. These data strengthen the hypothesis that Plasmodium utilizes melatonin to synchronize its life cycle, ${ }^{18,19,21}$ and, in the case of $P$. berghei and $P$. yoelii, we suggest that the non-response to melatonin is one of the reasons that this infection is unsynchronized.

While the physiology of malaria parasites seems similar, all displaying maturation stages such as ring, throphozoit, and schizont, the molecular machinery is distinct for different parasite strains. It is known that $80 \%$ of rodent malaria genes do possess an ortholog in P. falciparum. ${ }^{57}$

According to Guha and colleagues ${ }^{58}$ melatonin inhibits hepatocyte apoptosis and liver damage induced during malarial infection. In addition, $P$. berghei-infected hepatocytes are protected against apoptosis and this protection seems to be triggered by both host and parasite molecules. ${ }^{59}$ However, our results suggest that melatonin is not involved in hepatic infection by Plasmodium berghei ANKA sporozoites.

The molecular nature of the melatonin receptor in Plasmodia is currently under investigation. However, the complete absence of any functional effect of melatonin on $P$. berghei in vitro and its in vivo asynchronous development even in rats (that have a strong circadian melatonin production rhythm) suggests that this strain of Plasmodium does not express melatonin receptors.

Taken together these data add important novel support to the hypothesis that melatonin is responsible for the in vivo synchronicity of other Plasmodia species ${ }^{16,18-20}$ and suggest that the lack of response to melatonin is one of the reasons why the in vivo cell cycle of $P$. berghei and $P$. yoelii is unsynchronized.

Finally, the work presented here provides a clear link between the importance of host melatonin and synchronization of malaria parasites thus showing that the distribution of $P$. berghei and $P$. yoelii life forms is not affected by melatonin whereas, in contrast, it exerts a marked biological effect on $P$. chabaudi and P. falciparum.

\section{Acknowledgments}

We thank Fundação de Amparo à pesquisa de São Paulo (Fapesp) and CNPq for funding CRSG and Fundação para a Ciência e Tecnologia (FCT) of the Portuguese Ministry of Science (grant POCTI/SAU-MMO/60930/2004 to MMM. PB received fellowship from FAPESP. SE was supported by FCT fellowships (SFRH/BPD/31598/2006). We thank Dr Robert S Desowitz for the critical review and helpful suggestions.

\section{References}

1. Snow RW, Guerra CA, Noor AM, et al. The global distribution of clinical episodes of Plasmodium falciparum malaria. Nature. 2005;434:214-217.

2. Garcia CR, Takeuschi M, Yoshioka K, et al. Imaging Plasmodium falciparum-infected ghost and parasite by atomic force microscopy. J Struct Biol. 1997;119:92-98.

3. Przyborski JM, Lanzer M. Protein transport and trafficking in Plasmodium falciparum-infected erythrocytes. Parasitology. 2005;130:373-388.

4. Boyd GH. Induced variations in the asexual cycle of Plasmodium cathemerium. Am J Hyg. 1929;9:181-187.

5. Taliaferro WH, Taliaferro LG. Morphology, periodicity and course of infection of Plasmodium brasilianum in Panamanian monkeys. Am J Hyg. 1934;20:1-49.

6. Stauber LA. Factors influencing the asexual periodicity of avian malarias. Journal of Parasitology. 1939;95-116.

7. Hawking F. The clock of the malaria parasite. Sci Am. 1970;222:123-131.

8. Trager W, Jensen JB. Human malaria parasites in continuous culture. Science. 1976;193:673-675.

9. Pavithra SR, Banumathy G, Joy O, et al. Recurrent fever promotes Plasmodium falciparum development in human erythrocytes. $J$ Biol Chem. 2004;279:46692-46699.

10. Hawking F, Worms MJ, Gammage K. 24- and 48-hour cycles of malaria parasites in the blood; their purpose, production and control. Trans $R$ Soc Trop Med Hyg. 1968;62:731-765.

11. Garcia CR, Markus RP, Madeira L. Tertian and quartan fevers: temporal regulation in malarial infection. J Biol Rhythms. 2001;16:436-443.

12. Gambrell WE. Variations in gametocyte production in avian malaria. Am J Trop Med. 1937;17:689-727.

13. Hawking F, Worms MJ, Gammage K. Host temperature and control of 24-hour and 48-hour cycles in malaria parasites. Lancet. 1968; 1:506-509.

14. Gautret P, Motard A. Periodic infectivity of Plasmodium gametocytes to the vector. A review. Parasite. 1999;6:103-111.

15. Garcia CR, Markus RP, Madeira L. Tertian and quartan fevers: temporal regulation in malarial infection. J Biol Rhythms. 2001;5:436-443.

16. Vivien-Roels B, Pévet $P$, Claustrat B. Pineal and circulating melatonin rhythms in the box turtle, Terrapene carolina triunguis: effect of photoperiod, light pulse, and environmental temperature. Gen Comp Endocrinol. 1998;69(2):163-173.

17. Di Mascio P, Dewez B, Garcia CR. Ghost protein damage by peroxynitrite and its protection by melatonin. Braz J Med Biol Res. 2000;33:11-17.

18. Hotta CT, Gazarini ML, Beraldo FH, et al. Calcium-dependent modulation by melatonin of the circadian rhythm in malarial parasites. Nat Cell Biol. 2000;2:466-468. 
19. Gazarini ML, Thomas AP, Pozzan T, et al. Calcium signaling in a low calcium environment: how the intracellular malaria parasite solves the problem. J Cell Biol. 2003;161:103-110.

20. Beraldo FH, Garcia CR. Products of tryptophan catabolism induce $\mathrm{Ca}^{2+}$ release and modulate the cell cycle of Plasmodium falciparum malaria parasites. J Pineal Res. 2005;39:224-230.

21. Beraldo FH, Almeida FM, da Silva AM, et al. Cyclic AMP and calcium interplay as second messengers in melatonin-dependent regulation of Plasmodium falciparum cell cycle. J Cell Biol. 2005;170:551-557.

22. Ward P, Equinet L, Packer J, et al. Protein kinases of the human malaria parasite Plasmodium falciparum: the kinome of a divergent eukaryote. BMC Genomics. 2004;5:79.

23. Doerig C, Billker O, Pratt D, Endicott J. Protein kinases as targets for antimalarial intervention: Kinomics, structure-based design, transmission-blockade, and targeting host cell enzymes. Biochim Biophys Acta. 2005;1754;132-150.

24. Doerig C, Meijer L. Antimalarial drug discovery: targeting protein kinases. Expert Opin Ther Targets. 2007;11:279-290

25. Gardner MJ, Hall N, Fung E, et al. Genome sequence of the human malaria parasite Plasmodium falciparum. Nature. 2002;419:498-511.

26. Carlton JM, Angiuoli SV, Suh BB, et al. Genome sequence and comparative analysis of the model rodent malaria parasite Plasmodium yoelii yoelii. Nature. 2002;419:512-519.

27. Passos AP, Garcia CR. Characterization of $\mathrm{Ca}^{2+}$ transport activity associated with a non-mitochondrial calcium pool in the rodent malaria parasite P. chabaudi. Biochem Mol Biol Int. 1997;42:919-925.

28. Passos AP, Garcia CR. Inositol 1,4,5-trisphosphate induced $\mathrm{Ca}^{2+}$ release from chloroquine-sensitive and -insensitive intracellular stores in the intraerythrocytic stage of the malaria parasite P. chabaudi. Biochem Biophys Res Commun. 1998;245:155-160.

29. Docampo R, Moreno SN. The acidocalcisome. Mol Biochem Parasitol. 2001;114:151-159.

30. Kirk K. Membrane transport in the malaria-infected erythrocyte. Physiol Rev. 2001;81:495-537.

31. Farias SL, Gazarini ML, Melo RL, et al. Cysteine-protease activity elicited by $\mathrm{Ca} 2+$ stimulus in Plasmodium. Mol Biochem Parasitol. 2005;141:71-79.

32. Gazarini ML, Garcia CR. Interruption of the blood-stage cycle of the malaria parasite, Plasmodium chabaudi, by protein tyrosine kinase inhibitors. Braz J Med Biol Res. 2003;36:1465-1469.

33. Varotti FP, Beraldo FH, Gazarini ML, et al. Plasmodium falciparum malaria parasites display a THG-sensitive $\mathrm{Ca} 2+$ pool. Cell Calcium. 2003;33:137-144

34. Gazarini ML, Garcia CR. The malaria parasite mitochondrion senses cytosolic $\mathrm{Ca}^{2+}$ fluctuations. Biochem Biophys Res Commun. 2004;321:138-144.

35. Nagamune K, Sibley LD. Comparative genomic and phylogenetic analyses of calcium ATPases and calcium-regulated proteins in the apicomplexa. Mol Biol Evol. 2006;23:1613-1627.

36. Vaid A, Sharma P. PfPKB, a Protein Kinase B-like Enzyme from Plasmodium falciparum: II. Identification of calcium/calmodulin as its upstream activator and dissection of a novel signaling pathway. $J$ Biol Chem. 2006;281:27126-27133.

37. Lew VL, Tiffert T. Is invasion efficiency in malaria controlled by preinvasion events? Trends Parasitol. 2007;23:481-484.

38. Elliott R. The influence of vector behavior on malaria transmission. Am J Trop Med Hyg. 1972;21:755-763.

39. Das MK, Nagpal BN, Srivastava A, et al. Bioecology of An. philippinensis in Andaman group of islands. J Vector Borne Dis. 2003;40:43-48.
40. Wanji S, Tanke T, Atanga SN, et al. Anopheles species of the mount Cameroon region: biting habits, feeding behaviour and entomological inoculation rates. Trop Med Int Health. 2003;8:643-649.

41. Shililu J, Ghebremeskel T, Seulu F, et al. Seasonal abundance, vector behavior, and malaria parasite transmission in Eritrea. J Am Mosq Control Assoc. 2004;20:155-164.

42. Desowitz RS, Barnwell JW. Plasmodium berghei: deep vascular sequestration of young forms in the heart and kidney of the white rat. Ann Trop Med Parasitol. 1976;70:475-476.

43. Barnwell JW, Desowitz RS. Studies on parasitic crisis in malaria: I. Signs of impending crisis in Plasmodium berghei infections of the white rat. Ann Trop Med Parasitol. 1977;71:429-433.

44. Desowitz RS, Shida KK, Pang L, et al. Characterization of a model of malaria in the pregnant host: Plasmodium berghei in the white rat. Am J Trop Med Hyg. 1989;41:630-634.

45. Desowitz RS. Plasmodium berghei in the white rat: severe malaria of pregnancy does not occur in the progeny of mothers infected during gestation. Ann Trop Med Parasitol. 1999;93:415-417.

46. Hotta and colleagues. 2000.

47. Vivien-Roels B, Malan A, Rettori MC, Delagrange P, Jeanniot JP, Pévet P. Daily variations in pineal melatonin in inbread and outbread mice. J Biol Rhythms. 1998;13(5):403-409.

48. Di Virgilio F, Steinberg TH, Silverstein SC. Inhibition of Fura-2 sequestration and secretion with organic anion transport blockers. Cell Calcium. 1990;11:57-62.

49. Tsuji M, Mattei D, Nussenzweig RS, et al. Demonstration of heat-shock protein 70 in the sporozoite stage of malaria parasites. Parasitol Res. 1994;80:16-21.

50. Bruna-Romero O, Hafalla JC, Gonzalez-Aseguinolaza G, et al. Detection of malaria liver-stages in mice infected through the bite of a single Anopheles mosquito using a highly sensitive real-time PCR. Int J Parasitol. 2001;31:1499-1502.

51. Berridge MJ, Bootman MD, Roderick HL. Calcium signalling: dynamics, homeostasis and remodelling. Nat Rev Mol Cell Biol. 2003;4(7):517-529.

52. Thastrup O. Role of $\mathrm{Ca}^{2(+)}$-ATPases in regulation of cellular $\mathrm{Ca}^{2+}$ signalling, as studied with the selective microsomal $\mathrm{Ca}^{2(+)}$-ATPase inhibitor, thapsigargin. Agents Actions. 1990;29:8-15.

53. Lytton J, Westlin M, Hanley MR. Thapsigargin inhibits the sarcoplasmic or endoplasmic reticulum Ca-ATPase family of calcium pumps. $J$ Biol Chem. 1991;266:17067-17071.

54. Hearn J, Rayment N, Landon DN, et al. Immunopathology of cerebral malaria: morphological evidence of parasite sequestration in murine brain microvasculature. Infect Immun. 2000;68:5364-5376.

55. Billker O, Dechamps S, Tewari R, et al. Calcium and a calciumdependent protein kinase regulate gamete formation and mosquito transmission in a malaria parasite. Cell. 2004;117:503-514.

56. Marchesini N, Luo S, Rodrigues $\mathrm{CO}$, et al. Acidocalcisomes and a vacuolar $\mathrm{H}+$-pyrophosphatase in malaria parasites. Biochem $J$. 2000;(347 Pt 1):243-253

57. Hall N, Carlton J. Comparative genomics of malaria parasites. Curr Opin Genet Dev. 2005;15:609-613.

58. Guha M, Maity P, Choubey V, Mitra K, Reiter RJ, Bandyopadhyay U. Melatonin inhibits free radical-mediated mitochondrial-dependent hepatocyte apoptosis and liver damage induced during malarial infection. J Pineal Res. 2007;43(4):372-381.

59. Leiriao P, Mota MM, Rodriguez A. Apoptotic Plasmodium-infected hepatocytes provide antigens to liver dendritic cells. $J$ Infect Dis. 2005;191:1576-1581. 
